\title{
Why NOSQL And The Necessity of Movement Toward The NOSQL Data Base
}

\author{
Mostafa Omidi ${ }^{1}$, Mohsen Alipour ${ }^{2}$ \\ ${ }^{1,2}$ Technical Department, Azad University Branch of Tehran, Center
}

\begin{abstract}
The increasing development of substructures, services and applied programs, non-same structure of Data and other problems caused the classic databases sometimes can't response the human demands in huge data saving domain. Therefore, the NOSQL technology has been generated to response two main demand and problem in databases, A: increasing the data volume and demand for complicated saving structure and B: Lack of accountability in relational databases.
\end{abstract}

Keywords: Data Base, SQL, NOSQL, Big Data, Query, Data Adapter

\section{Introduction}

Big Data is an expression of huge and varied Data, come from numerous sources. It forecasted that this kind of Data growing $40 \%$ annually and has reached to zettabyte. Most of them have text structure or do not have structure. Considering the Data increasing speed, the most of applications need to be answered by operators and otherwise the opportunities will be lost.

NoSQL technology is a good choice in using of this kind of Data and can be a good substituent for Relational Database. It should be considered that NoSQL could not be always the substituent of Relational Database. It is indeed a parallel technology with Relational Database.

\section{Classic Databases}

Classic database is a collection of saved data upon to a distinct data model, which are in relationship to each other not only as physical form but also in other forms at least logical. They can be used by a concentrated system management and be utilized by one or more operators in share form simultaneously. They have table structure and their governing rules are based on ACID. Some examples of this kind are MS SQL Server, IBM DB2and Oracle that use the standard Questioning language its name is SQL.

\section{NoSQL Database}

The NoSQL expression is a general name, given to databases that don not use the SQL questioning. This database has some especial properties that do not exist in Relational databases. For example, the data in NoSQL can be saved without any limiting pattern definition. NoSQL has the taking Query ability from several thousand distributed servers. In addition, it has the Cache option in memory for reduction of delay time and increasing the efficiency. NoSQL databases has the BASE (Basically Available, Soft State, Eventual consistency) property. This kind of database are generally available and maybe be unavailable because of very especial disaster. For example, the GFS (Google File System) can be named which has the highest availability. This kind of database provides a view environment for the data using that increases the efficiency and speed of availability. In addition, these databases have high adaptability, for instance, when a new storage is added to network it begins the data transferring to the other storages rapidly.

\section{Types of NoSQL database}

NoSQL databases have different types that mainly divided into four category.

- Key/value Databases: In this method, the Data have a key value that the way of that Data achieving is that key. In this method, the keys are indexed; therefore, the speed of reading and writing on it is high.

- Document Oriented Databases: in this model, the amounts are saved as a structured and identifiable in a document. One of the benefits of this method is that we do not need key else and the amount of documents can be indexed. All related information of an operator is accessible via a Query and it is efficient for content oriented applications. This kind of databases save the documents in XML-Yandel-JSON format; MangoDB-CouchDB is kind of such databases. 


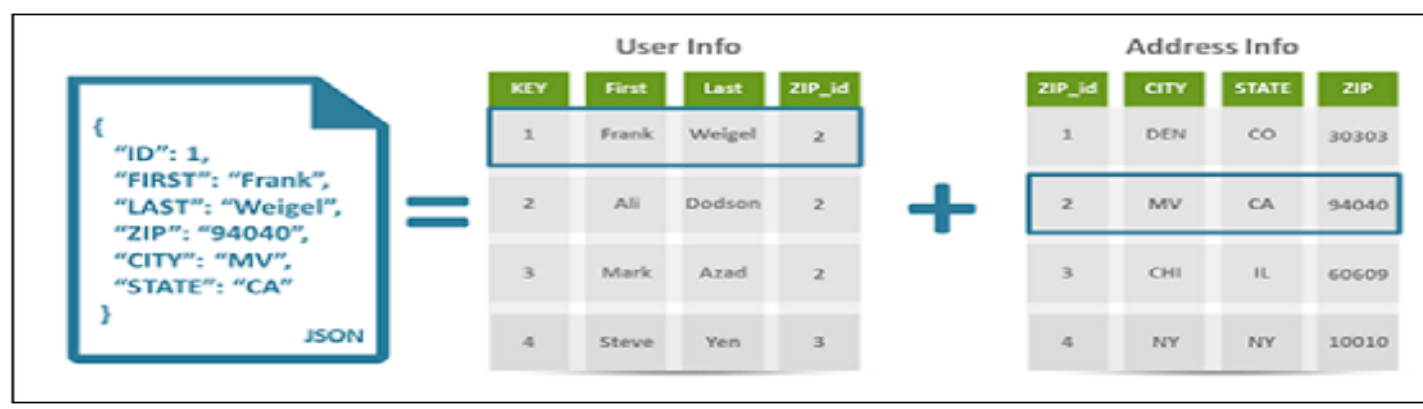

Fig1

- Stored ordered column-oriented databases: they are used for saving and processing huge amount of distributed data on several machines. In this method also used keys but the keys pointed to some tables and categorizes to different column family.In other words, it is a table, divided into some columns. In this method, information saved regular and it helps for information accessibility. For example, a person has different information in various field and in each three-group columns of personal information has a key. Hbase-Cloudta is an example of this kind.

- Graph Databases: components of this database are nodes and their base come from graph and use flexible graph structure instead of tables consist of rows and columns. This kind of databases are suitable for applications that infinite components relate to each other. Some applications such as social communications, transportation, road maps etc. Neo4j-FlockDB is one of the most famous databases.

Data adapters as a link between Relational databases and NOSQL

As it said before, because of the increasing development of Data that force to use NOSQL, andmany Relational database exist nowadays; therefore this technology introduced as data adapter.

\section{Used components in Data adapter}

1. Relational database

2. No SQL database

3. DB Adapter

4. DB Convertor

\section{Data Adapter Performance Way}

Practically the data adapter system is an interface between the applicable programs and database and is the responsible among the layers. When a command reaches to DB convertor at first, for accomplishment, the data adapter decides to command runs in relational database or NOSQL.

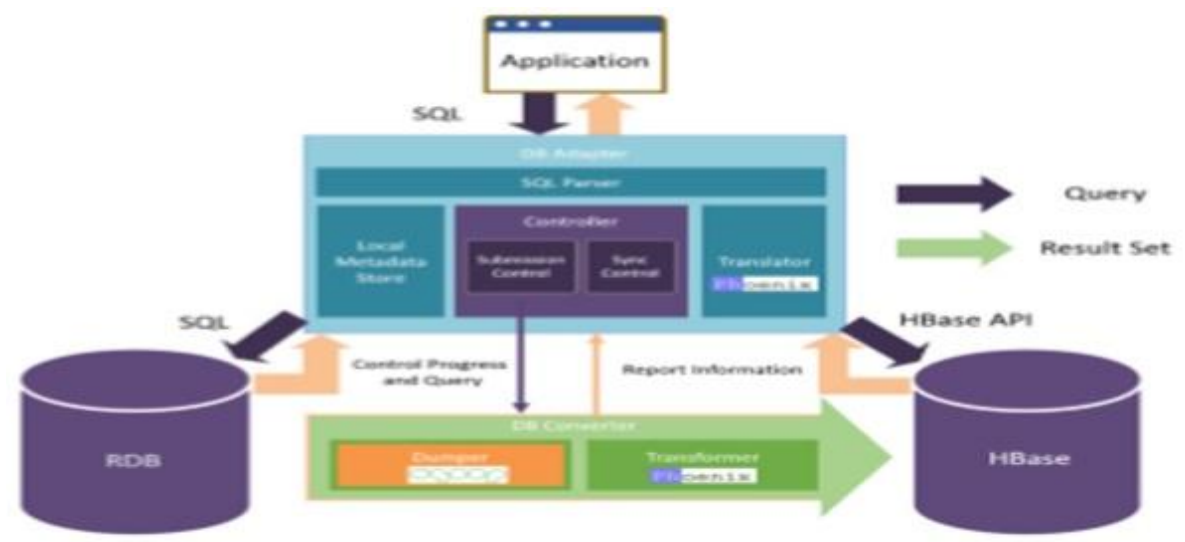

Fig2. Data Adapter

The most important productions, presented in NOSQL

Validity of NOSQL database projects can be suitable criteria for abilities and reasonable Support of a database.

- Dynamo: has performed by Amazon and it is the most considerable key-value NOSQL database. Amazon needed a scalable distributed platform to response the commercial activities in extra dimensions.

- Cassandra: it is a project, presented by Facebook as open text and is a key-value NOSQL. It written in Java form and used by Facebook and twitter. 
- $\quad$ Big Table: it is a column oriented and it is the Google property particularly.

- $\quad \mathrm{H}$ base: it is a database for random reading and writing. This distributed database that modelled and run from the Google Big Table. In addition, it has the saving ability in enormous volume. Yahoo and Facebook also use this database.

- Mango DB: it is a document-oriented database. Its development and design has done by 10gen Company. GitHub is one of the most important users of that.

- $\quad$ Neo4j: Use for social network analyze to their graph theory upon an open text graph database.

\section{Comparing the database selection criteria}

- Database programming language: it can increases the efficiency of database.

- Software license: An official document determines the using way and its duplication.

- Query language: simplicity is important when the query language is the interface between the database and operator.

- Initial publication year: it can help database to reach in maturity and is a criteria of selecting the database.

- $\quad$ Secondary Index: it provides the ability of other field indexing.

- Transactions: it is a collection of database operational works that should be done completely or backs to initial state when a problem occurs. This criterion is an important ability for sensitive systems such as the banks.

- The best use: it shows that database operates better than other databases in what situation.

- Educational information: amount of document and educational information accessibility of database known as an important factor in database using.

\section{References}

[1] Handling-Big-Data-Using-NoSQL report.2015.19-IEEE

[2] Data-adapter-for-querying-and-transformation-between-SQL-and-NoSQL-database report 23 July 2015

[3] BASE-analysis-of-NoSQL-database report J. future.2015.05.03

[4] "MongoDB, MySQL Compare Grid MongoDB. [Online]. Available: http://www.mongodb.org/display/DOCS/MongoDB,+CouchDB,+MySQL+Compare+Grid. [Accessed: 14-Jan-2013] 